\title{
Fuzzy Sets and Astrotheology
}

\author{
PAUL T E CUSACK*
}

Independent Researcher, BSc E, DULE, 1641 Sandy Point Rd, Saint John, NB, Canada E2K 5E8, Canada

*Corresponding Author: PAUL T E CUSACK, Independent Researcher, BSc E, DULE, 1641 Sandy

Point Rd, Saint John, NB, Canada E2K 5E8, Canada

Abstract: In this paper, we make use of fuzzy set theory as applied to the Game of Life Physics. We will see that fuzzy sets in combination with the Game of Life Physics provides us with the solution to the problem of how DNA, the human Brain and the universe interact.

Keywords: Game of Life Physics; AT Math; Fuzzy Sets; Cosmology; DNA; Brain

\section{INTRODUCTION}

The Game of Life Physics provides us with rules:

There is only one rule in the Game of Life Physics. It is:

For each cell in the grid, count how many of its 8 neighbours are ON at the present instant. If the answer is exactly two, that cell stays in its present state (ON or OFF) in the next instant. If the answer is exactly three, the cell is ON in the next instant whatever its current state. Under all other conditions, the cell is OFF. [1]

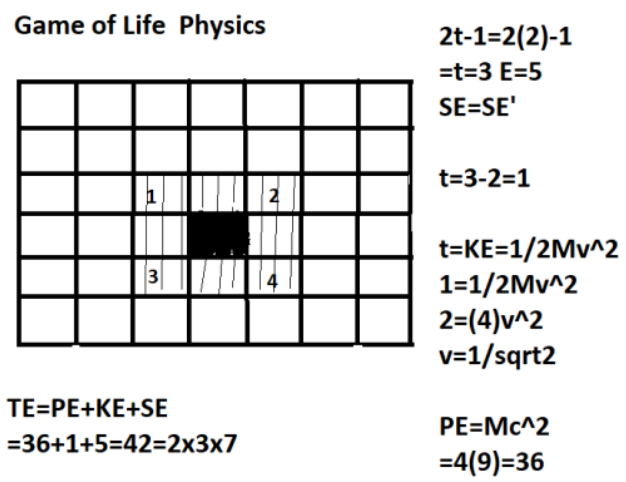

Figure1. Game of Life Physics Board

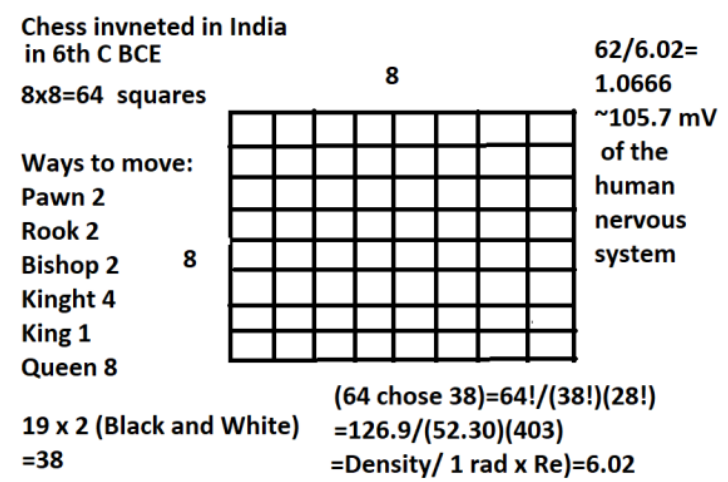

Figure2. Chess Board 
The first part gives us a fuzzy set:

$\{8$ choose 2$\}=8 ! /\{2 !(6 !)=28 \Rightarrow 1$ Or 0

$\{\{8$ choose 3$\}=1$

$28(1)+56(1)=84$

$28(0)+56(1)=56$

$56(0)=0$

$\{8$ choose 2$\}=$ fuzzy Set 0 or 1

$0 !=1$ !

$1=1$

True

$28(0 !)+56(1)=84$

$28(1)+56(1)=84$

$\mathrm{C}(\mathrm{A})=\operatorname{Core}(\mathrm{A})=\mathrm{A}^{1}=\{\mathrm{x} \in \mathrm{U} \mid \mathrm{m}(\mathrm{x})=1\}=\{28 \varepsilon 84 \mid \mathrm{m}(\mathrm{x})=1\} \quad 28 / 84=0.333=1 / \mathrm{c}$

$\mathrm{S}(\mathrm{A})=\operatorname{Supp}(\mathrm{A})=\mathrm{A}^{>0}=\{\mathrm{x} \varepsilon \mathrm{U} \mid \mathrm{m}(\mathrm{x})>0\}=\{56 \varepsilon 84 \mid \mathrm{m}(\mathrm{x})>0\} \quad 56 / 84=0, .666=\mathrm{G}$

$\mathrm{A}^{1}=\left\{\begin{array}{ll}28 & 56\end{array}\right\}^{1}$

$\mathrm{A}^{>0}=\left\{\begin{array}{ll}56 & 84\end{array}\right\}$

$\mathrm{A}^{>0}-\mathrm{A}^{1}=\left\{\begin{array}{ll}56 & 84\end{array}\right\}-\left\{\begin{array}{ll}28 & 56\end{array}\right\}=\left\{\begin{array}{ll}28 & 28\end{array}\right\}=28\left\{\begin{array}{ll}1 & 1\end{array}\right\}$

$(\mathrm{U}, \mathrm{m})^{>0}-(\mathrm{U}, \mathrm{m})^{1}=28\left\{\begin{array}{ll}1 & 1\end{array}\right\}$

$\left\{\begin{array}{ll}84 & 1\end{array}\right]-(84>0)=28\left\{\begin{array}{ll}1 & 1\end{array}\right\}$

$\{84-84,1->0)=28\{1,1\}$

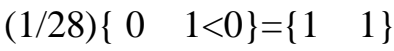

$\left\{\begin{array}{ll}0 \quad(1 / 28)<0\}=\chi\{1 & 1\end{array}\right\}$

$\{0, \quad 0.0357<0\}=\chi\left\{\begin{array}{ll}1 & 1\end{array}\right\}$

$\chi(1)=0 \quad \chi 0.0357=1$

$\chi=0 \quad \chi=1 / 0.0357=28$

$\mathrm{A}=\left\{\begin{array}{ll}0 & 28\end{array}\right\}$

$=28\left\{\begin{array}{ll}0 & 1\end{array}\right\}$

$=28 \mathrm{I}$

$\{8$ choose 2$\}=28=1$ Or 0 Fuzzy Set

$\mathrm{C}(\mathrm{A})=\operatorname{Core}(\mathrm{A})=\mathrm{A}^{1}=\{\mathrm{x} \in \mathrm{U} \mid \mathrm{m}(\mathrm{x})=1\}=\{28 \varepsilon 84 \mid \mathrm{m}(\mathrm{x})=1\} \quad 28 / 84=0.333=\mathbf{1} / \mathrm{c}$

$\mathrm{S}(\mathrm{A})=\operatorname{Supp}(\mathrm{A})=\mathrm{A}^{>0}=\{\mathrm{x} \varepsilon \mathrm{U} \mid \mathrm{m}(\mathrm{x})>0\}=\{56 \varepsilon 84 \mid \mathrm{m}(\mathrm{x})>0\} \quad 56 / 84=0, .666=\mathbf{G}$

$\mathrm{A}^{1}=\{0,28\}=28\{0,1\}=28 \mathrm{~L}$

$\mathrm{A}^{>0}=\left\{\begin{array}{ll}56 & , 84\end{array}\right\}^{0}=\mathrm{G}\left\{\begin{array}{ll}84 & 12\end{array}\right\}$

$=\mathrm{G}=1 / 28=28 \mathrm{G}=1$

$(28(0.666)=186.648=1 / 536$

2. Golden Mean Parabola

$\mathrm{t}^{2}-\mathrm{t}-1=\mathrm{E}$

536²-536-1=1.0507 105 mV (Human Nervous System)

$\mathrm{V}=\mathrm{iR}$

International Journal of Scientific and Innovative Mathematical Research (IJSIMR) 
$105=(1 / \mathrm{c}) \mathrm{R}$

$\mathrm{R}=3.15$

\section{Brain:}

We know $y=m x+b=1 / 2 t+2$

$\mathrm{y}=1 / 2 \mathrm{t}+2$

$=28+2=30$

$=10 \mathrm{c}$

$\mathbf{t}=\mathbf{3 0}$

$\mathrm{y}=1 / 2 \mathrm{t}+2$

$=1 / 2(28)+2$

$=14+2=16$

$16^{2}+16-2=270$

$=10 \mathrm{c}^{3}$

$\mathrm{t}=270 ; \mathrm{E}=16$

$\mathbf{t}=\mathbf{3} ; \mathrm{E}=5$

$\mathrm{t}=23 \mathrm{E}=11$

$\mathrm{t}=\mathrm{E}^{2}+\mathrm{E}-2$

$=11^{2}+11-2$

$=13$

$\mathrm{E}=13^{2}+13-2$

$=180=\pi$

$E=t^{2}-t-1$

$24^{2}-24-1=551$

$\mathrm{t}=1 / \mathrm{E}=1 / 181 \sim 1 / 180=1 / \pi$

$\mathrm{s}=|\mathrm{E}| \mathrm{t} \mid \sin 60$

$=0.866$

$=\mathrm{t}$

$t=\sin 60$

$\mathrm{t}=\sin \theta$

$\left.\sin ^{-1} 0.84\right)=57.14$

$57.14 \times 3.15 / 180=1 \mathrm{rad}=\mathrm{t} \quad \theta=\pi / 2$

\section{Human DNA}

$2.15 \times 1.67$ Dalton $=\mathbf{3 6 . 5 5} \mathbf{g m}$

$\mathrm{M}=\mathrm{Ln} \mathrm{t}$

$\mathrm{M}=\mathrm{Ln} \mathrm{t}$

$36.55=\mathrm{Ln} \mathrm{t}$

$\mathrm{t}=747$

$\mathrm{E}=1 / \mathrm{t}=0.1338 \sim \mathrm{s}$

$\mathrm{s}=\mathrm{Et} \sin 60$ 
$\mathrm{s}=\mathrm{t}$

$\mathrm{E}=1 / \sin 60$

$\mathrm{E}=1 / \sin 60^{\circ}$

$\mathrm{s}=\mathrm{E} \times \mathrm{t}=|\mathrm{E} \| \mathrm{t}| \sin 60^{\circ}$

$\mathrm{s}=\left(1 / \sin 60^{\circ}\right) \mathrm{t}\left(\sin 60^{\circ}\right)$

$\mathrm{s}=\mathrm{t}$

\section{Universe:}

Universal Parametric Equation:

$\left[\csc 60^{\circ} ; 3\right]=\{\mathrm{E}, \mathrm{t}]$

$\mathrm{E}=1 / \sin 60^{\circ}=1 / \mathrm{t}=\mathrm{E}$

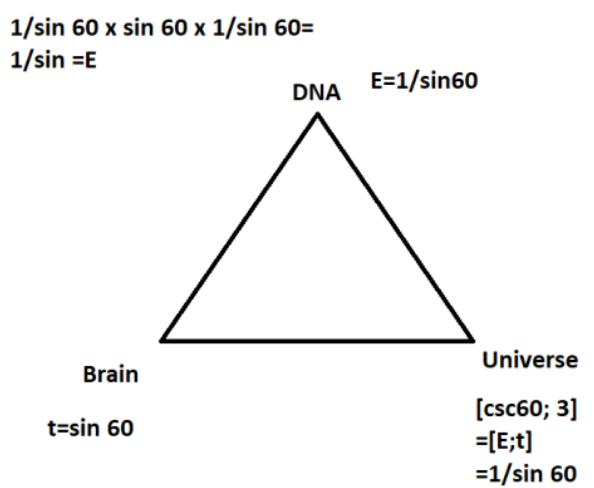

\section{CONCLUSION}

We see that fuzzy sets in combination with the Game of Life Physics provides us with the solution to the problem of how DNA, the human Brain and the universe interact.

\section{REFERENCES}

[1] Cusack, PTE., Game of Life Physics; Chess, and AT Math (Submitted)

Citation: PAUL T E CUSACK, Fuzzy Sets and Astrotheology, International Journal of Scientific and Innovative Mathematical Research (IJSIMR), vol. 8, no. 8, pp. 22-25, 2020. Available : DOI: https://doi.org/10.20431/ 2347-3142.0808003

Copyright: (C) 2020 Authors. This is an open-access article distributed under the terms of the Creative Commons Attribution License, which permits unrestricted use, distribution, and reproduction in any medium, provided the original author and source are credited. 